\title{
A Low-Cost Scalable Matched Filter Bank Receiver for GFSK Signals with Carrier Frequency and Modulation Index Offset Compensation
}

\author{
Charles Tibenderana and Stephan Weiss \\ Communications Research Group \\ School of Electronics \& Computer Science \\ University of Southampton, UK \\ \{ct02r,s.weiss\}@ecs.soton.ac.uk
}

\begin{abstract}
This paper addresses a low-cost matched filter bank (MFB) receiver for GFSK signals with estimation and correction of carrier frequency and modulation index offsets, such as found in Bluetooth systems. The proposed MFB implementation exploits redundancies in an otherwise prohibitively large system of filter banks by performing matched filtering over a symbol period followed by an iterative process of phase propagation. The resulting scheme has low complexity, is scalable, and permits the estimation of carrier frequency and modulation index offsets from the intermediate MFB outputs. We present simulations and a comparison of the proposed system for a Bluetooth scenario.
\end{abstract}

\section{Introduction}

Gaussian Frequency Shift Keying (GFSK) is a bandwidth preserving digital modulation technique used for low-cost transmission standards like Bluetooth [1]. The simple and inexpensive receiver functionality of Bluetooth can be enhanced if the excess computational capabilities of a common hardware platform shared with more complex wireless local area network standards, such as proposed in some software defined radio systems, are exploited [2].

High performing receivers for continuous phase modulated signals, of which GFSK is a subset, include the Viterbi and the matched filter bank (MFB) receivers [3]. However, unlike the MFB [4, 5], the Viterbi [6] receiver requires a rational and precisely known modulation index (h) [7], and neither of these conditions is guaranteed in Bluetooth [1]. We have derived a low-cost, scalable realisation of the MFB receiver, using a smaller set of intermediate filters, followed by an iterative phase propagation process [8]. This will enable operation at a much lower channel signal to noise ratio than popular methods like FM-AM conversion, phase-shift discrimination, zero-crossing detection, or frequency feedback would allow [9, 10], but without the prohibitive complexity of the conventional MFB receiver.

For the MFB receiver operation over hostile channels, in the past we have successfully applied blind equalisation to mitigate dispersiveness [11]. Carrier frequency offsets were targeted in $[8,11]$ based on the receiver input. In this paper, we propose new schemes for carrier frequency and modulation index offset correction for the efficient MFB receiver implementation. The proposed schemes utilise intermediate filter outputs for offset estimation, and regeneration of the smaller set of intermediate filter coefficients for correction, thereby overcoming performance deterioration which would otherwise occur in systems such as Bluetooth $[12,7]$.

The structure of this paper is as follows: To begin a system model is developed in Sec. 2. In Sec. 3 we highlight the operation of the standard MFB receiver for GFSK signals, before the efficient realisation of the MFB is derived. Sec. 4 contains proposals for carrier frequency, modulation index, and timing synchronisation. Finally simulation results are presented in Sec. 5, before concluding in Sec. 6 .

\section{System Model}

GFSK generally modulates a multilevel symbol stream $p[k]$, which here is assumed to be binary, $p[k] \in\{ \pm 1\}$ with bit index $k$. This bit sequence is expanded by a factor of $N$ and passed through a Gaussian filter with a bandwidthtime product $K_{B T}$ and impulse response $g[n]$ of length $L N$, thus having a support of $L$ bit periods, yielding its discrete instantaneous angular frequency signal

$$
\omega[n]=2 \pi h \sum_{k=-\infty}^{\infty} p[k] g[n-k N],
$$

where $n$ represents the chip index, while $h$ is the modulation index. The phase of the baseband version of the transmitted signal,

$$
s[n]=\exp \left\{j \sum_{\nu=-\infty}^{n} \omega[\nu]\right\}=\prod_{\nu=-\infty}^{n} e^{j \omega[\nu]},
$$

is determined as the cumulative sum over all previous frequency values $\omega[n]$. 
We assume that the received signal $r[n]$ is subjected to a carrier frequency $\Omega$ and additive white Gaussian noise (AWGN) $v[n]$, which is uncorrelated with $s[n]$, such that

$$
r[n]=s[n] e^{j \Omega n}+v[n]
$$

Since a dispersive channel can be sufficiently well equalised [11], its effect has been neglected in (3).

\section{$3 \quad$ MFB Receiver}

\subsection{Standard Receiver}

A standard MFB receiver is based on a filter bank containing all possible transmitted sequences $s_{i, j}[n]$ over a duration of $K$ bit periods, where $s_{i, j}[n]$ are the legitimate transmitted sequences with $i \in\{ \pm 1\}$ indicating the value of the middle bit and $j=0(1) 2^{K+L-2}$ indexing the possible combinations of the remaining $K-1$ bits. The detection determines the output bit according to

$$
\hat{p}[k]=\arg \max _{i}\left|\sum_{n=0}^{K N-1} r[k N-n] \cdot s_{i, j}^{*}[-n]\right| \quad,
$$

and achieves near-optimum maximum likelihood noncoherent estimation of a GFSK symbol in AWGN [4, 5, $13,3,14]$. Note that the detector imposes a delay such that ideally $\hat{p}[k]=p\left[k-\frac{K+1}{2}\right]$.

As a result of the support length of the Gaussian filter, over the $K$ bit observation interval $2^{K+L-1}$ legitimate signal sequences exist if we neglect the initial phase shift. Therefore, a computational complexity of

$$
C_{\text {standard }}=2 N K 2^{K+L-1}=N K 2^{K+L}
$$

real valued multiply accumulates (MACs) arises. The cost in (5) consider that the possible sequences $s_{i, j}[n]$ consist of complex conjugate pairs, and that a complex valued operation accounts for 4 real valued ones. Nevertheless, the complexity in (5) is prohibitive. Therefore, in the Sec. 3.2 we describe a low complexity implementation of this receiver.

\subsection{Iterative Receiver}

The matched filter responses $s_{i, j}^{*}[-n]$ in Sec. 3.1 are designed from the transmitted signal $s[n]$ in (2). In the following, we summarise the approach in [8] to obtain a low cost iterative implementation of the MFB.

The MFB outputs over a single bit period, $K=1$, can be calculated as

$$
\mathbf{y}_{k}^{(1)}=\mathbf{W}^{(1)} \mathbf{r}_{k}
$$

where $\mathbf{r}_{k}$ represents the received signal samples $r[n]$ held in a length $N$ tap delay line, and $\mathbf{W}^{(1)} \in \mathbb{C}^{2^{L} \times N}$ contains the $2^{L}$ possible complex conjugated transmitted waveforms in its rows, and represents an intermediate filter bank. For the example of $L=3$, the phase trajectories of the resulting 8 waveforms are shown in Fig. 1. The superscript ${ }^{(1)}$ in (6) indicates that only a single bit period $K=1$ is observed.

For $K>1$, the possible phase trajectories can be derived from the case $K-1$ by augmentation of the curves shown in Fig. 1 and the application of appropriate phase shifts. This allows us to formulate iteratively for $\mathbf{y}_{k}^{(K)} \in \mathbb{C}^{2^{K+L-1}}$ [8]

$$
\mathbf{y}_{k}^{(K)}=\mathbf{D}^{(K)} \mathbf{A}^{(K)} \mathbf{y}_{k}^{(K-1)}+\mathbf{M}^{(K)} \mathbf{y}_{k-K+1}^{(1)},
$$

where

$$
\begin{aligned}
& \mathbf{M}^{(K)}=\left[\begin{array}{l}
\mathbf{M}^{(K-1)} \\
\mathbf{M}^{(K-1)}
\end{array}\right] \text { with } \mathbf{M}^{(1)}=\mathbf{I}_{2^{L}} \\
& \mathbf{A}^{(K)}=\left[\begin{array}{lr}
\mathbf{A}^{(K-1)} & \mathbf{0} \\
\mathbf{0} & \mathbf{A}^{(K-1)}
\end{array}\right] \quad \text { with } \\
& \mathbf{A}^{(1)}=\text { blockdiag }\left\{\left[\begin{array}{l}
1 \\
1
\end{array}\right]\right\} \in \mathbb{Z}^{2^{L} \times 2^{L-1}} \\
& \mathbf{D}^{(K)=}\left[\begin{array}{lr}
\mathbf{D}^{(K-1)} & \mathbf{0} \\
\mathbf{0} & \mathbf{D}^{(K-1)}
\end{array}\right] \text { with } \mathbf{D}^{(1)}=\operatorname{diag}\{\mathbf{w}\} .
\end{aligned}
$$

The vector $\mathbf{w}$ in (7) contains the first column of $\mathbf{W}^{(1)}$, i.e. the final phase rotations after one bit period as shown in Fig. 1. This form of the MFB receiver is depicted by the flow graph in Fig. 3. A detector similar to (4), selecting the index of the largest element would operate on $\mathbf{y}_{k}^{(K)}$ to determine the correct output bit.

\subsection{Computational Complexity}

Inspecting the operations in Fig. 3, each bit period $2^{L}$ matched filter operations of length $N$ have to be performed. With the matrices $\mathbf{M}^{(k)}$ and $\mathbf{A}^{(k)}$ only performing indexing, the only arithmetic operations required are multiplications with the diagonal elements of the phase correction

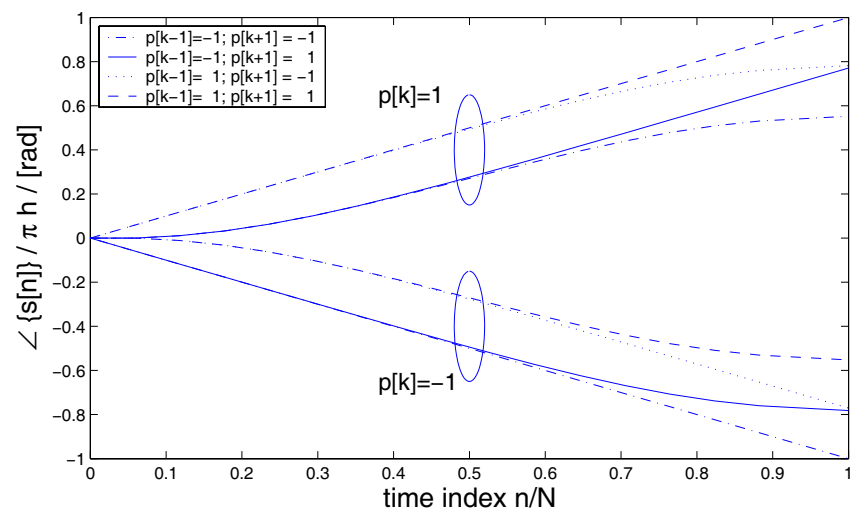

Fig. 1. Example of the legitimate phase trajectories of $s[n]$ for one bit period when $K_{B T}=0.5, L=3, h=0.35$ and an initial phase of zero. 


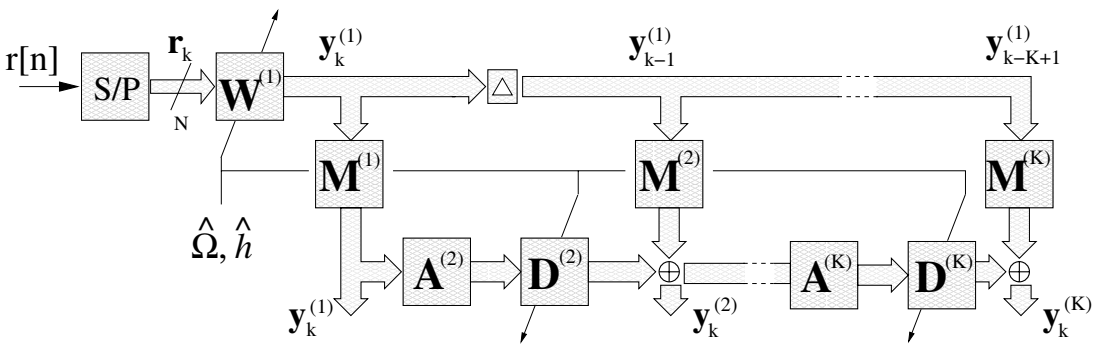

Fig. 3. Low-complexity implementation of a matched filter bank high-performance GFSK receiver. The received GFSK signal $r[n]$ is passed through a serial/parallel converter and a smaller intermediate filter bank $\mathbf{W}^{(1)}$ with a single bit duration. Processed over $K$ stages, the matched filter bank outputs are contained in $\mathbf{y}_{k}^{(K)}$.

matrices $\mathbf{D}^{(K)}$, yielding a total of

$$
C_{\text {efficient }}=4 \cdot 2^{L} N+4 \sum_{k=1}^{K-1} 2^{L+k} \lesssim 4 \cdot 2^{L}\left(N+2^{K-1}\right)
$$

While the functionality of the MFBs in Secs. 3.1 and 3.2 are identical, their computational complexities according to (5) and (8) differ considerably, as evident from Fig. 2. Particularly for long observation intervals and high sample resolution, the low-cost approach offers a viable route for a high performance MFB receiver.

\section{Synchronisation}

In this section we assume that the parameters for carrier frequency and modulation index in the receiver, $\hat{\Omega}$ and $\hat{h}$ respectively, are not accurately known and need to be estimated. The methods proposed below are based on intermediate MFB outputs, and adjustment of $\hat{\Omega}$ and $\hat{h}$ affects intermediate $\mathbf{W}^{(1)}$ in (6) and subsequently $\mathbf{D}^{(k)}$ in (7), which is indicated in Fig. 3. Timing synchronisation is not detailed below, but can be achieved in the maximum likelihood sense by techniques described in [3].

\subsection{Carrier Frequency}

By excluding the noise term in (3), such that

$$
r[n]=s[n] e^{j \Omega n}
$$

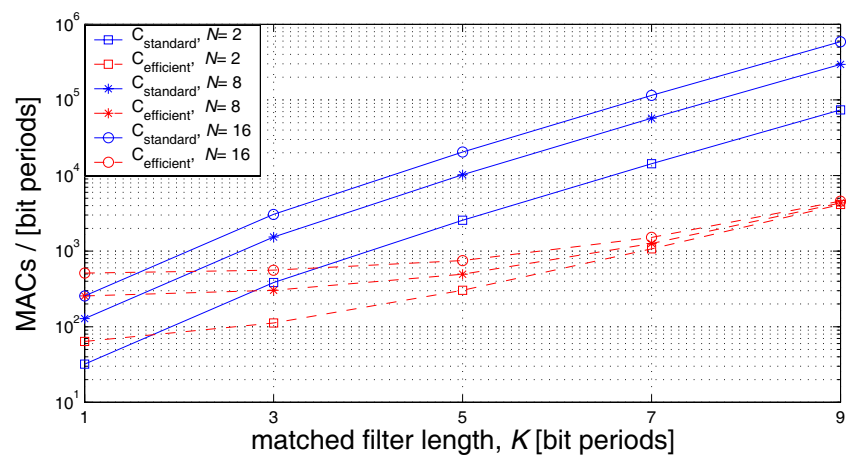

Fig. 2. Complexity comparison: standard vs. efficient MFB receiver for binary GFSK. it is obvious that $\angle \mathcal{E}\{r[n]\} \propto \Omega$, and therefore the offset $\Delta \Omega=\Omega-\hat{\Omega}$ causes a difference in the phase trajectories computed by the transmitter and receiver. Thus, the phase term

$$
\eta=\angle \mathcal{E}\left\{\left(y_{k-(K-1) / 2}^{(1)} \cdot\left(y_{k-(K-3) / 2}^{(1)}\right)^{*}\right)\right\} \propto \Delta \Omega
$$

can be verified to be proportional to the mismatch in carrier frequency $\Delta \Omega$. In (9), the quantity $y_{k}^{(1)}$ refers to the element of $\mathbf{y}_{k}^{(1)}$ in Fig. 3, associated with the correct bit sequence leading to the detection of the middle bit $p\left[k-\frac{K+1}{2}\right]$. The complex conjugate term in (9) ensures that the phase is measured relative to zero.

Due to (9), the receiver carrier frequency estimate $\hat{\Omega}$ can be adjusted by

$$
\hat{\Omega}[k+1]=\hat{\Omega}[k]+\mu_{\Omega} \cdot \hat{\eta}[k],
$$

where $\hat{\eta}[k]$ is an instantaneous estimate of the term in (9) based on a single bit period,

$$
\hat{\eta}[k]=\angle\left\{y_{k-(K-1) / 2}^{(1)} \cdot\left(y_{k-(K-3) / 2}^{(1)}\right)^{*}\right\} .
$$

Note that in (10), the element $y_{k}^{(1)}$ is based on the estimated bit sequence rather than the true quantities assumed in (9). Since $y_{k}^{(1)}$ is available from the proposed low-cost MFB, the only additional complexity arises from $2^{L} N$ MACs for modifying $\mathbf{W}^{(1)}$ and consequently $\mathbf{D}^{(k)}$.

\subsection{Modulation Index}

Observation of (1) and (2) reveals that $|\angle\{s[n]\}| \propto h$. For the example of possible phase trajectories of $s[n]$ in Fig. 1, assuming that the phase at the beginning of the bit period was zero, an increase in $h$ would cause the phase trajectories to fan out further. Since both the transmitted signal and $\mathbf{W}^{(1)}$ are derived from this set of curves, the phase term

$$
\chi=\angle \mathcal{E}\left\{\left(y_{k-(K-1) / 2}^{(1)} \cdot\left(y_{k-(K-3) / 2}^{(1)}\right)^{*}\right) \cdot p\left[k-\frac{K+1}{2}\right]\right\} \propto \Delta h
$$

can be verified to be proportional to the mismatch in modulation index between the transmitter and receiver, $\Delta h=h-\hat{h}$. Analogous to Sec. 4.1, in (11), the quantity $y_{k}^{(1)}$ refers to the element of $\mathbf{y}_{k}^{(1)}$ in Fig. 3, associated 
with the correct bit sequence leading to the detection of the middle bit $p\left[k-\frac{K+1}{2}\right]$. The complex conjugate term in (11) ensures that the phase is measured relative to zero, while $p\left[k-\frac{K+1}{2}\right]$ compensates for the sign change imposed by the middle bit onto the phase as evidenced in Fig. 1.

To adapt the modulation index estimate $\hat{h}$ and therefore $\mathbf{W}^{(1)}$ and $\mathbf{D}^{(k)}$ in the receiver, we employ an iterative technique

$$
\hat{h}[k+1]=\hat{h}[k]+\mu_{h} \cdot \hat{\chi}[k],
$$

where $\hat{\chi}[k]$ is an instantaneous estimate of the term in (11) based on a single bit period,

$$
\hat{\chi}[k]=\angle\left\{y_{k-(K-1) / 2}^{(1)} \cdot\left(y_{k-(K-3) / 2}^{(1)}\right)^{*}\right\} \cdot \hat{p}[k]
$$

Note that in (13), the element $y_{k}^{(1)}$ is based on the estimated bit sequence and the estimated middle bit $\hat{p}[k]$ rather than the true quantities assumed in (11). The adaptation of $\hat{h}[k]$ according to (12) requires few extra computations since the coefficients in $\mathbf{W}^{(1)}$ and subsequently $\mathbf{D}^{(k)}$ can be adjusted together with the carrier frequency offset estimate at the same time.

\section{Simulation Results}

In our experiments we employed system parameters $K_{B T}=$ $0.5, h=0.35$, in order to simulate a Bluetooth signal, and $N=2$. Where applicable $\Delta \Omega$ and $\Delta h$ are set to magnitudes of $\frac{2 \pi 75}{N \cdot 1000}$ radians and 0.07 respectively, to represent the maximum normalised offsets permitted between a communicating transceiver pair adhering to the Bluetooth standard. The required system performance is a bit error ratio (BER) of at most $10^{-3}$, which is the maximum allowed in Bluetooth [1].

The runtime of the efficient algorithm was a fraction of that of the standard MFB receiver simulator, which is expected from the results portrayed in Fig. 2. Despite this, the BER of the two realisations are equivalent and depicted in Fig. 4 for various values of $K$. Performance improves significantly with an increase in $K$, up to $K \approx 9$, where $10^{-3}$ BER is achieved at $9.8 \mathrm{~dB}$ Eb/No.

The BER plots in Fig. 5 suggest that an MFB system with $K=9$ and an uncompensated modulation index offset of $\Delta h=0.07$ results in $3 \mathrm{~dB}$ loss, while an uncompensated carrier frequency offset of $\Delta \Omega=0.075 \pi$ causes the system to collapse. It was noted that frequency offset and modulation index errors cause an accumulation in phase error, which amplifies the problem for larger observation intervals. Figs. 5 and 6 demonstrate the efficacy of the carrier frequency and modulation index offset correction algorithms. In Fig. 6, it takes less than 500 iterations for convergence of $\hat{\Omega}$ and $\hat{h}$ when $\mu_{\Omega}=\mu_{h}=0.01$ and $K=5$. As exemplified in Fig. 5, the algorithms restore ideal performance in AWGN. However, it must be noted that for low $\mathrm{Eb} / \mathrm{No}, \mu_{\Omega} \gg \mu_{h}$ for convergence to occur.

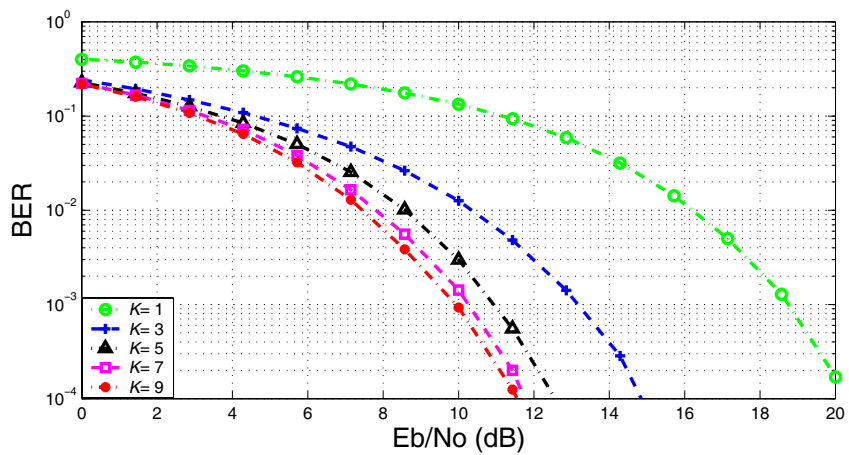

Fig. 4. BER performance in AWGN with different observation intervals $K$, and $K_{B T}=0.5, h=0.35$.

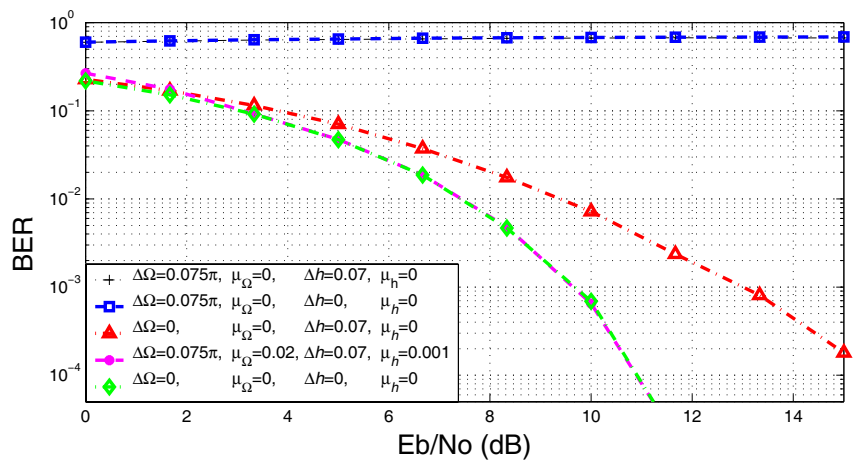

Fig. 5. BER performance improvement due to carrier frequency and modulation index correction algorithms with $K_{B T}=0.5, h=0.35, N=2$, and $K=9$.

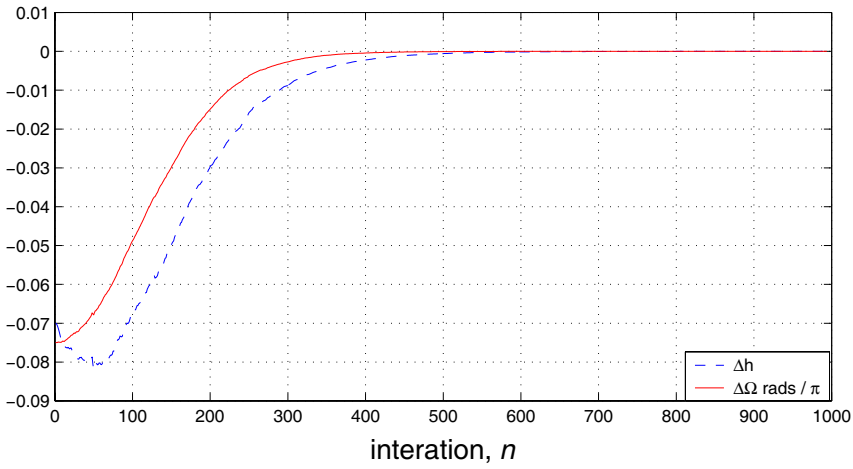

Fig. 6. Learning curves for simultaneous carrier frequency and modulation index offset correction when $K_{B T}=0.5$, $h=0.35, N=2, K=5, \Delta \Omega=-\frac{2 \pi 75}{N \cdot 1000}, \Delta h=-0.07$, and $\mu_{\Omega}=\mu_{h}=0.01$.

\section{Conclusion}

With the advent of SDR, simple wireless standards like Bluetooth, which employs GFSK will have extra computational capacity if sharing a hardware platform with a more complex one. We suggest an efficient implementation of a matched filter bank receiver as a potential use of this extra resource. Our realisation of the MFB saves approx- 
imately $90 \%$ complexity for a 9 bit observation interval, and maintains the near optimal BER performance of the standard MFB receiver. In addition, we have shown that outputs from the smaller set of intermediate filters used in the efficient realisation provide a means to detect offsets in carrier frequency and modulation index, both of which degrade reception of GFSK signals, and should be expected in networks like Bluetooth where nontrivial offsets in carrier frequency and modulation index are permitted. Such offsets can then be corrected for by adjusting the coefficients of the intermediate filter coefficients, and thus adding negligible complexity to the filter bank. The offset correction algorithms are fast and do not require any training.

\section{References}

[1] Bluetooth Special Interest Group, Specification of the Bluetooth System, February 2002, Core.

[2] Roel Schiphorst, Fokke W. Hoeksema, and C.H. Slump, "A (Simplified) Bluetooth Maximum A Posteriori Probability (MAP) Receiver," in Proc. IEEE Workshop on Signal Processing Advances in Wireless Communications, Rome, Italy, June 2003.

[3] John B. Anderson, Tor Aulin, and Carl-Erik Sundberg, Digital Phase Modulation, Plenum Press, New York and London, 1986.

[4] William P. Osborne and Micheal B. Luntz, "Coherent and Noncoherent Detection of CPFSK," in IEEE Transactions on Communications, August 1974, vol. COM-22, pp. 10231036.

[5] Thomas A. Schonhoff, "Symbol Error Probabilities for Mary CPFSK: Coherent and Noncoherent Detection," in IEEE Transactions on Communications, June 1976, vol. COM-24, pp. 644-652.
[6] Tor Aulin, Nils Rydbeck, and Carl-Erik W. Sundberg, "Continous Phase Modulation-Part II: Partial Response Signaling," IEEE Transactions on Communications, vol. COM-29, no. 3, pp. 210-225, March 1981.

[7] Amir Soltanian and Robert E. Van Dyck, "Performance of the Bluetooth System in Fading Dispersive Channels and Interference," in Proc. Global Telecommunications Conference, San Antonio, Texas, November 2001, vol. 6, pp. 3499-3503.

[8] Charles Tibenderana and Stephan Weiss, "LowComplexity High-Performance GFSK Receiver With Carrier Frequency Offset Correction," in Proc. IEEE International Conference on Acoustics, Speech, and Signal Processing, Montreal, Canada, May 2004, vol. IV, pp. 933-936.

[9] Bruce A. Carlson, Communication Systems, McGraw-Hill, Singapore, 3rd edition, 1986.

[10] Roel Schiphorst, Fokke Hoeksema, and Kees Slump, "Bluetooth Demodulation Algorithms and their Performance," in Proc. 2nd Karlsruhe Workshop on Software Radios, Karlsruhe, March 2002, pp. 99-105.

[11] Charles Tibenderana and Stephan Weiss, "Blind Equalisation and Carrier Offset Compensation for Bluetooth Signals," in European Signal Processing Conference, Vienna, Austria, September 2004, pp. 909-912.

[12] Craig Robinson, Alan Purvis, Armin Lechner, and Michael Hoy, "Characterisation of Bluetooth Carrier Frequency Errors," in Proc. IEEE Mixed Signal Testing Workshop, June, Ed., Seville, Spain, June 2003, pp. 119-124.

[13] W. Hirt and S. Pasupathy, "Suboptimal Reception of Binary CPSK Signals," in Proc. IEE Communications, Radar and Signal Processing, United Kingdom, June 1981, vol. 128, pp. 125-134.

[14] John G. Proakis, Digital Communications, McGraw-Hill, New York, 3rd edition, 1995. 